\title{
POTENCIALIDADES, ALCANCES Y LIMITACIONES DEL CURSO DE LICENCIATURA EN EDUCACIÓN DEL CAMPO DE LA UNIVERSIDAD FEDERAL DO RECÔNCAVO DA BAHIA PARA LA MOVILIZACIÓN DEL CONOCIMIENTO Y DESARROLLO SOCIAL DE LAS COMUNIDADES CAMPESINAS DE LA CIUDAD DE LAJE-BAHIA-BRASIL.
}

\author{
POTENCIALIDADES, ALCANCES E LIMITAÇÕES DO CURSO DE LICENCIATURA DE \\ EDUCAÇÃO DO CAMPO DA UNIVERSIDADE FEDERAL DO RECÔNCAVO DA BAHIA PARA A \\ MOVIMENTAÇÃO DO CONHECIMENTO E DESENVOLVIMENTO SOCIAL DAS COMUNIDADES \\ CAMPONESAS DA CIDADE DE LAJE-BAHIA-BRASIL.
}

André B. Sandes Judith Naidorf ${ }^{2}$

\begin{abstract}
Brasil es un país que posee dimensiones continentales y con gran parte del territorio disponible para la práctica de actividades agropecuarias. Contrastando con esta realidad, innúmeras personas que viven de la tierra, nunca tuvieron condiciones de poseerla, y los pequeños campesinos enfrentan grandes dificultades de mantenerla y resistir a las presiones del agro negocio. Discutir esas cuestiones desde la perspectiva del campesino es de gran relevancia, de modo que puedan cuestionar el modelo hegemónico vigente, organizarse políticamente y crear alternativas propias y eficientes para minimizar las desigualdades sociales existentes, que son el resultado acumulativo de las desventajas transmitidas a través de generaciones. Basado en una investigación bibliográfica, análisis documental y entrevistas con estudiantes del curso de Educación del Campo de la UFRB que viven en la ciudad de Laje-Bahia-Brasil, este trabajo contribuirá para una mejor comprensión de la función social de la Universidad. De esta forma, serán presentados los resultados de un estudio que tuvo como objetivo principal comprender las potencialidades, alcances y limitaciones del curso en cuestión y cómo los graduandos desarrollan sus actividades, movilizan el conocimiento entre esos dos universos (universidad $x$ comunidad) y contribuyen para el desarrollo social colectivo. Es a través de una educación emancipadora que el ejercicio de la ciudadanía se ampliará, contribuyendo en el proceso de formación de personas capaces de percibirse como protagonistas de la historia y atentas a las cuestiones inmediatas que repercuten en su vida y de la comunidad donde viven.
\end{abstract}

Palabras clave: Universo Rural. Educación del Campo. Universidad. Movilización del conocimiento.

O Brasil é um país que possui dimensões continentais e com grande parte do território disponível para a prática de atividades agropecuárias. Contrastando com esta realidade, inúmeras pessoas que vivem da terra nunca tiveram condições de possui-la e os pequenos camponeses enfrentam grandes dificuldades de mantê-la e resistir às pressões do agro negocio. Discutir essas questões desde a perspectiva do camponês é de grande relevância, de modo que possam questionar o modelo hegemónico vigente, organizar-se politicamente e criar alternativas próprias e eficientes para minimizar as desigualdades sociais existentes, que são o resultado acumulativo das desvantagens transmitidas através de gerações. Baseado em uma investigação bibliográfica, análise documental e entrevistas com estudantes do curso de Educação do Campo da UFRB que vivem na cidade de Laje-Bahia-Brasil, este trabalho contribuirá para uma melhor compreensão da função social da universidade. Desta forma, serão apresentados os resultados de um estudo que teve como objetivo principal compreender as potencialidades, alcances e limitações do curso em questão, e como os graduandos desenvolvem suas atividades, movimentam o conhecimento entre esses dois universos (universidade $x$ comunidade) e contribuem para o desenvolvimento social coletivo. É através de uma educação emancipadora que o exercício da cidadania se ampliará, contribuindo no processo de formação de pessoas capazes de perceber-se como protagonistas da historia e atentas para as questões imediatas que repercutem em sua vida e da comunidade onde vivem.

Palavras-chave: Universo Rural. Educação do Campo. Universidade. Movimentação do conhecimento.

\footnotetext{
${ }^{1}$ Licenciado em Geografia e Pedagogia, Especialista em Educação Ambiental y Gestão Educacional, Mestre em Teologia - Educação Comunitária com Infância e Juventude (EST), Doutor em Educação (UCSF), Professor Regente pela Secretaria de Educação do Estado da Bahia-Brasil e Coordenador Pedagógico pela SEC-Laje (Bahia-Brasil). Membro do Grupo de pesquisa PANACUM - Grupo de Estudo e Pesquisa em Física e Ensino de Ciências (UFRB) e Estrategias de movilización del conocimiento en Ciencias Sociales y Humanidades (UBA-Argentina) coordenado por la Doctora Judith Naidorf. Ese trabajo es producto de la estancia posdoctoral en Ciencias Humanas y Sociales de la Universidad Nacional de Buenos Aires -Argentina. absandes@hotmail.com. CV: http://lattes.cnpq.br/0920106898724426

${ }^{2}$ Licenciada em Ciências da Educação. Doutora da Universidade de Buenos Aires. Docente da Faculdade de Filosofia e Letras, UBA. Pesquisadora Adjunta de CONICET no Instituto de Pesquisa em Ciências da Educação da Faculdade de Filosofia e Letras da Universidade de Buenos Aires, Argentina. judithnaidorf@gmail.com.
} 


\section{INTRODUCCIÓN}

La Universidade Federal do Recôncavo da Bahia (UFRB ${ }^{3}$ ) está ubicada en la ciudad de Amargosa (Recôncavo Sul da Bahia - Brasil) y fue creada el 29 de julio de 2005, constituida en un modelo multicampo que posee unidades instaladas en las ciudades de Cruz das Almas, Amargosa, Cachoeira, Feira de Santana, Santo Amaro y Santo Antônio de Jesus.

De acuerdo con Santos (2018, p. 02), el grado de Educación del Campo es ofrecido en el Centro de Formación de Profesores (CFP) de Amargosa y tuvo inicio a fines de 2013. El referido CFP poseía en abril de 2018 un total de 138 docentes, 53 servidores técnicos, 51 empleados tercerizados y atendía a un total de 1.584 estudiantes.

El curso en cuestión posee una propuesta distinta al de las carreras convencionales, aunque su duración sea también de cuatro años. Las actividades académicas son divididas en dos períodos distintos y complementarios: $80 \%$ del curso es en "Tiempo Universidad" (TU) durante el cual los estudiantes tienen acceso a los conocimientos sistematizados, y los $20 \%$ restantes son en "Tiempo Comunidad" (TC), cuando realizan actividades en contacto directo con la realidad campesina, previamente planeadas, orientadas y acompañadas por los docentes de la UFRB.

Esa propuesta, que concilia encuentros presenciales y alternancia, permite el diálogo entre estos dos universos, contribuyendo así a mejorar la realidad de los campesinos y, paralelamente, ampliar la mirada da la universidad para su contexto inmediato. De esta forma, tiene estrecha relación con la categoría de movilización del conocimiento ${ }^{4}$, una vez que pone la ciencia a servicio de la sociedad y orienta sus agendas a la resolución de problemas locales, estrechando así los lazos entre universidad, investigadores, estudiantes y los conocimientos tradicionales de las comunidades. Formar una red integrada de intercambio entre esos sujetos es un gran reto.

Este trabajo tiene como objetivo principal comprender mejor cómo ocurre esta interacción, así como las potencialidades, alcances y limitaciones del grado de Educación del Campo en lo que se refiere a la categoría de movilización del conocimiento y desarrollo social de las comunidades campesinas de la ciudad de Laje - Bahía - Brasil.

El interés por ese tema se justifica, porque es relativamente nuevo y por no haber investigaciones que aborden las contribuciones de este curso en el contexto específico. Partimos de dos hipótesis: 1 . El diálogo entre universidad y campesinos, a través de los estudiantes del curso de Educación del Campo es posible y necesario para mejorar la calidad de vida de las personas que viven de la tierra. 2 . El curso de Educación del Campo de la UFRB posee una propuesta contra hegemónica comprometida con la movilización del conocimiento y con el desarrollo de las comunidades campesinas de la región del Recôncavo Sul da Bahia, entre ellas la zona rural de la ciudad de Laje.

Comprender la contribución de esta institución en el contexto regional, la utilidad y aplicabilidad del conocimiento producido y compartido con los estudiantes, así como el retorno que ellos llevan para el lugar donde realizan su trabajo cotidiano es, sin duda, una contribución importante para ajustar los planeamientos futuros de la institución y orientar los trabajos realizados por los estudiantes en el Tiempo Comunidad.

\footnotetext{
'La UFRB fue creada por la Ley 11.151 de 29 de julio de 2005. Posee 7 unidades denominadas 'Centros de Enseñanza', instalados en las ciudades de Cruz das Almas (que posee 2 centros y es donde está la Rectoría), Amargosa, Cachoeira, Feira de Santana, Santo Amaro y Santo Antonio de Jesus. Ofrece actualmente 46 cursos de grado, siendo 14 en licenciaturas; y 25 cursos de postgrado, siendo 2 doctorados, 13 maestrías (siendo 8 académicas y 5 profesionales), 8 especializaciones y 2 residencias médicas. Al final de 2017 (semestre 2017.1 ), la UFRB contaba con 8.545 estudiantes, siendo 7.717 en cursos de grado y 997 de postgrado y poseía un total de 906 docentes, 711 servidores técnicos, además de servidores tercerizados y pasantes (SANTOS, 2018, p. 01).

${ }^{4}$ En este escenario, a partir del año 2000, surge para la discusión de las políticas de investigación en Ciencias Sociales el concepto de movilidad o movilización del conocimiento (knowledge mobilization); esto es, el requerimiento de una producción de conocimiento "listo para la acción", que implica ir más allá de su difusión, en tanto también sería función del investigador encontrar caminos que enlacen la producción y la utilización del conocimiento producido. Sin embargo, dado que se han identificado dificultades en los canales de diálogo entre productores y usuarios de los conocimientos científicos, esta propuesta de movilidad o movilización del conocimiento es acompañada por lo que se denomina "interlocutores/gestores del conocimiento" o knowledge brokers, que adquieren la forma de promotores/articuladores o matchmakers (Naidorf, Vasen y Alonso, 2016, p. 10).
} 
Basado en una investigación bibliográfica, análisis documental de la institución en cuestión y entrevistas con estudiantes que hicieron el curso de Licenciatura en Educación del Campo (UFRB), este trabajo procurará contribuir a una mejor comprensión de la función social de la universidad y de la resolución de problemas concretos relacionados a la vida campesina.

\section{Potencialidades, alcances y limitaciones del curso de Licenciatura en Educación del Campo en las comunidades campesinas de la ciudad de Laje-Bahia-Brasil}

A pesar de las conquistas y avances ocurridos en los últimos años, aún hay mucho por hacer para consolidar y ampliar los resultados positivos, de modo que todas las personas logren tener acceso a las mismas oportunidades de desarrollo.

Este cambio involucra a varios actores sociales, sin embargo, aquellos relacionados a la educación, sea formal o de cualquier categoría, son indispensables para un cambio más profundo y duradero. Cabe aquí citar a Paulo Freire (2000, p. 59), "[...] si la educación sola no transforma a la sociedad, sin ella tampoco la sociedad cambia". Dermeval Saviani (2003, p. 93) también demuestra concordar con eso al afirmar que "[...] la educación interfiere sobre la sociedad, pudiendo contribuir para su propia transformación”. A su vez, Itana Barreto (2012) apunta que la emancipación de actores sociales, "[...] está intrínsicamente vinculada a los procesos pedagógicos transparentes y de empoderamiento individual y colectivo para favorecer la participación por la autonomía, criticidad y la acción transformadora", (Barreto, 2012, p. 20).

Partiendo del presupuesto de que las universidades son espacios privilegiados de apropiación, construcción y difusión de conocimiento, de investigación y extensión, de diálogo y debate de interés colectivo y, sobre todo, de centros de apoyo a las causas sociales, es importante evaluar su contribución, alcances y límites para que sigan atendiendo a las expectativas de la sociedad, especialmente de aquellos grupos marginalizados o excluidos por el sistema vigente.

Es de conocimiento de todos que hay pocos cursos universitarios que se predisponen a investigar la realidad concreta con el rigor que la ciencia exige y, paralelamente, comprometerse con la movilización del conocimiento para la resolución de problemas concretos que permitan un cambio substancial.

Es justamente en ese escenario que se piensa la categoría de movilización del conocimiento que, según Naidorf y Perrotta (2015, p.4) posee:

[...] una riqueza reflexiva en tanto que nos motiva a enlazar la necesidad de una ciencia social orientada - epistemológica, metodológica y ontológicamente - al cambio social en aquellas sociedades periféricas, desiguales y dependientes (ciencia social politizada) con el requerimiento de que ese tipo de conocimiento de lo social pueda, al mismo tiempo, tener un componente preocupado por su impacto efectivo (ciencia social móvil).

El intercambio universidad y comunidad orientado a la búsqueda de alternativas viables para mejorar la vida de las personas con conocimientos científicos y tecnológicos es un camino posible y necesario. Es importante subrayar que "[...] no se trata ya de volcar hacia la sociedad las soluciones acabadas, sino de la necesidad de estudiar los problemas y avanzar en la búsqueda de soluciones con las mismas poblaciones con las que se trabaja" (NAIDORF, 2009, p. 195). Según Naidorf (2009, p. 196), es necesario "[...] desarrollar políticas hacia la sociedad, romper con el aislamiento y convertir la educación superior pública en un sistema activo que se enmarque en los cambios y necesidades políticas, económicas y sociales de la sociedad".

El curso de Educación del Campo ofrecido en la UFRB demuestra esta preocupación con el panorama social de una región geográfica de Brasil, marginalizada y con el menor Índice de Desarrollo Humano (IDH) de este país (NAIDORF; SANDES, 2019, p. 52). Para demostrar ese compromiso, tomaremos como objeto de investigación la ciudad de Laje, específicamente los estudiantes del referido curso, que son los responsables directos por ese diálogo entre universidad y comunidad. 
Laje es una pequeña ciudad, ubicada en Recôncavo Sul da Bahia (latitud1310'58.5" sur y longitud $39^{\circ} 25^{\prime} 32.61 "$ oeste), que se encuentra distante aproximadamente $230 \mathrm{Km}$ o $135 \mathrm{Km}$ (vía ferry boat) de Salvador, capital del estado de Bahía y que hace frontera con Mutuípe, Ubaíra, Santo Antônio de Jesus, Aratuípe, Valença, Jiquiriçá, São Miguel das Matas y Amargosa.

Como todos los lugares de la faja costera de Brasil, en el municipio en cuestión predominaba la vegetación de mata atlántica en el período colonial, donde vivían aborígenes pertenecientes a los grupos Tupiniquim y Tupinaé, que con el pasar del tiempo fueron exterminados o expulsos de su tierra para dar lugar a las haciendas que producian caña de azúcar, café, tabaco y aguardiente (especie de bebida alcohólica producida a base de caña en los ingenios de la región), que formaban la base de la riqueza en el período colonial.

Actualmente posee una población aproximada de 22 mil personas, mayoritariamente ubicada en la zona rural (16 mil personas viven en el campo, equivalente a $73 \%$ del total) compuesta por 54 comunidades y tres distritos, donde hay 18 asociaciones comunitarias registradas y otras en proceso de formación (IBGE, 2010). Es importante subrayar que Laje es el típico caso de ciudad donde el concepto de rural y urbano se confunden, ya que las sedes de los municipios mantienen una fuerte relación con la tierra.

Con IDH medio de 0,586 (IBGE, 2010), su economía es básicamente agrícola, con producción expresiva de productos derivados de la mandioca y con una pequeña producción de naranja, banana y cacao. Su pecuaria es diversificada, predominando creación de bovinos. Por encontrarse en un valle, cuyo río principal es el Jiquiriçá, su sitio urbano se presenta bastante desnivelado, con laderas que poseen declividad relativamente grande para los patrones de la región y calles estrechas (SANDES, 2019, p. 35).

Para el desarrollo de este trabajo, fueron realizadas, además de investigación bibliográfica, observación de las actividades desarrolladas por ocho estudiantes que están concluyendo el curso de Educación de Campo en la UFRB, análisis de relatos de experiencias e informes presentados a la universidad, entrevistas individuales y ruedas de conversación con estudiantes y miembros de las comunidades campesinas.

Para tanto, se utilizó el método fenomenológico, que consiste en describir la experiencia tal como es, y el método observacional, que consiste en observar y registrar eventos relevantes relacionados con el tema de la investigación y enriquecidos con relatos de experiencias de los estudiantes. Fueron realizados innúmeros encuentros, ruedas de conversación e invitaciones, a fin de conocer la forma de trabajo y las intervenciones realizadas en los años que hicieron el curso y realizaron sus actividades en la comunidad.

Provocar una reflexión sobre la práctica y los resultados da las intervenciones en la comunidad es fundamental para encontrar las alternativas más apropiadas para lograr éxito junto a los campesinos. Algunas declaraciones, registradas abajo, demuestran muy bien los resultados del trabajo de diálogo realizado con y por los estudiantes de la UFRB en su contexto social.

1. Mediar una rueda de conversación no es ser dueño del saber, y sí darle oportunidad a las personas, compartir conocimientos y construir caminos, por medio de intercambios de experiencia, porque que todos somos aprendices (Declaración de un campesino que participó de un trabajo realizado por Josimario Marques en una comunidad de quilombo).

2. A través de esa experiencia fue posible reflexionar sobre nuestra postura como futuros educadores del campo, sobre las cuestiones inherentes al ejercicio de la docencia, perfeccionamiento de nuestras prácticas y la posibilidad de proponer discusiones que valoricen a los sujetos del campo y reafirmen su identidad y cultura en las diversas localidades. Sentimos el piso de la escuela en clase, conociendo sus potencialidades y debilidades. En síntesis, vivimos la 
realidad de los profesores y profesoras que ejercen en ese embate y que continúan luchando por una educación contextualizada para que las comunidades hagan parte de esa construcción de sujetos emancipados, de sujetos de derecho. (Registro de un estudiante del curso de Educación del Campo de la UFRB).

3. En Laje, es notorio el protagonismo de las asociaciones en las acciones sociales comunitarias. Eso causó impactos positivos en las comunidades locales, esas intervenciones, protagonizadas por la organización, han promovido la elaboración de proyectos y empoderamiento comunitario que influencia en la calidad de vida de los campesinos. (Registro de un estudiante del curso de Educación del Campo de la UFRB).

4. El trabajo realizado junto a las asociaciones se convierte en un espacio de estudio y aprendizaje. Eso demuestra que la educación está más allá de las paredes de la universidad, está en los espacios de reuniones, en la convivencia cotidiana y en las acciones realizadas en las comunidades rurales. Allí surge un líder, una persona que, aunque muchas veces sin oportunidad de estudiar, se interesa por las cuestiones del campo y en participar de la asociación de su comunidad. (Registro de un estudiante del curso de Educación del Campo de la UFRB).

5. Muchas comunidades comprendieron la importancia de organizarse donde viven y varios jóvenes, padres y madres de familia, voluntariamente, ponen su vida a servicio del bien colectivo por el simple deseo de ver a su comunidad organizada. Para eso es necesario unirse a otras asociaciones de comunidades vecinas. La lucha nace de las necesidades del pueblo, que aprende en la escuela que la convivencia es la garantía de una vida digna para todos". (Registro de un estudiante del curso de Educación del Campo de la UFRB).

Como se puede observar, la reflexión sobre la práctica es indispensable tanto para los sujetos que viven en el campo como para el futuro educador que quiere realizar un buen trabajo. Según Freitas (2000, p. 205):

La concepción de profesor como profesional "práctico reflexivo" se desarrolla en oposición a la concepción de profesor percibido como técnico, que limita sus necesidades a la adquisición de contenidos, métodos y técnicas, en una postura meramente aplicativa y reproductora. De otra forma, en el abordaje denominado "profesor reflexivo", la actividad educativa del profesor es concebida como actividad compleja que no siempre puede ser desarrollada a partir de procedimientos previamente organizados, exigiendo del profesor una postura reflexiva para crear alternativas de enseñanza.

Para Freire, el acto de investigar no está disociado del acto de enseñar. Esa idea está explícita cuando afirma: "Hace parte de la naturaleza de la práctica docente la indagación, la búsqueda, la investigación [...] enseño porque busco, porque indago, porque indago y me indago" (Freire, 1996, p. 32). Así, distanciarse epistemológicamente de su práctica para poder mejor desempeñar su papel de educar, aproxima la filosofía a la realidad, atribuyendo sentido a las actividades desarrolladas en el cotidiano y reflexionando sobre sus resultados con más profundidad.

Aunque el hábito de registrar no sea muy común entre los educadores, ese acto se configura como un importante instrumento de reflexión sobre la práctica. De esta forma, los informes disponibles 
ayudaron a comprender la naturaleza de las actividades en Tiempo Comunidad. A seguir, serán compartidas algunas consideraciones al respecto de los relatos de educadores del campo y otros aspectos observados durante el proceso investigativo en la práctica.

\section{En relación al perfil de los estudiantes investigados}

- Mayoritariamente son mujeres con edad entre veintitrés y treinta años, residentes en la zona rural de la ciudad de Laje, donde realizaron las actividades e intervenciones planeadas para el Tiempo Comunidad exigido por la UFRB.

- Hijos de padres agricultores, de renta mensual baja, que viven de la tierra, cuyas familias están formadas por 3 a 5 miembros, configurándose como familia pequeña en relación a las décadas anteriores.

- Les gusta vivir en el campo y se relacionan bien con las personas de la comunidad. Todos demostraron poseer una relación de pertenencia e identidad con el lugar, se sienten apoyados y responsables en contribuir para mejorar la calidad de vida de su pueblo, y el curso de la UFRB fortaleció aún más ese lazo con la tierra y la comunidad.

\section{Trayectoria de estudio}

- Los estudiantes investigados fueron a la escuela pública en la zona rural, donde completaron la educación primaria $\left(1^{\circ}\right.$ a $5^{\circ}$ año). Después fueron a la ciudad para dar continuidad a los estudios, ya que no había escuelas en su región para los años subsecuentes.

- Tuvieron un aprovechamiento regular en la enseñanza básica; algunos se justificaron diciendo que los maestros no estaban preparados para trabajar en ese medio, no traían al aula materiales apropiados que valoricen al hombre del campo, su cultura y particularidades.

- Conocieron el curso de Educación del Campo a través de amigos que ya habían estudiado en la UFRB y ven en la universidad la posibilidad de cambiar de vida, ayudar a su familia y a la comunidad como un todo.

- Recibieron algún tipo de auxilio para estudiar (becas de auxilio y permanencia en la universidad) y algunos participaron de la residencia pedagógica, que es un programa remunerado por el gobierno para actuar en escuelas del campo. Se trata de una gran oportunidad para mejorar el currículo, adquirir experiencia, crear identidad docente, aprender en la práctica lo que estudió en la UFRB y ayudar en la formación de niños y niñas campesinas.

- Muchos de los estudiantes dijeron que eran los primeros de la familia en conseguir nivel académico y, en la propia comunidad, en tener acceso a la universidad pública.

- Les gusta el curso y lo recomiendan para otras personas que viven en ambientes rurales, se interesan por la educación y por la dinámica de los campesinos.

- Los estudiantes quieren dar continuidad a la vida académica, haciendo una complementación en un curso de licenciatura en el área de humanas, especializarse y de ser posible, hacer la maestría en Educación del Campo ofrecido por la propia UFRB.

- En el futuro les gustaría actuar en el área de educación formal en el campo.

3 En relación al curso de Educación del Campo de la UFRB y el Tiempo Universidad

- Ayudó a comprender mejor la comunidad, sus dificultades y la importancia de participar activamente, organizarse políticamente para conseguir visibilidad y garantizar los derechos logrados con mucha lucha.

- Evaluaron positivamente el curso en cuestión, aunque reconocieron lagunas en la matriz curricular, falta de preparación de algunos profesores para trabajar con ese público específico (no vienen de la Educación del Campo ni hacen parte del grupo de investigación de esa área, adoptando así, metodologías convencionales y evaluaciones selectivas). Ausencia de disciplinas que profundicen los contenidos fundamentales relacionados a la educación como Sociología de la Educación, Historia de la Educación y también que los ayuden a trabajar con pecuaria, común en el medio rural. 
- Consideraron interesante la propuesta de Tiempo Comunidad y Tiempo Universidad y la realización de las etapas exigidas por el curso: observación, coparticipación y regencia.

- Hicieron críticas a la supervisión de los profesores de la UFRB que, según ellos, fueron muy exigentes, pero no correspondieron con el apoyo necesario. En contrapartida, los profesores justificaron su ausencia por falta de estructura logística de transporte para acompañar las actividades. De esta forma, el acompañamiento por el supervisor fue básicamente a distancia y en talleres promovidos en la universidad.

4 En relación a las experiencias en las prácticas de educación formal

- Las prácticas se realizaron en tríos para discutir temáticas relacionadas a identidad campesina y políticas públicas del campo, línea del tiempo y avances en la agricultura familiar, fuentes de energía alternativas, técnicas posibles de aplicar en la práctica, medio ambiente, entre otras temáticas relacionadas a la vida campesina.

- Las prácticas en la educación básica fueron realizadas en área urbana, pero en escuelas que atienden mayoritariamente estudiantes oriundos de la zona rural. Los estudiantes universitarios manifestaron que pasaron a tener otra visión de la escuela, muy distinta a la que tenían cuando ellos mismos eran alumnos.

- Los estudiantes afirmaron haber sido bien recibidos por los directores y profesores de las escuelas y que lo que aprendieron con los profesores regentes contribuyó en su proceso de formación.

- Los alumnos también los recibieron con respeto y participaron de las clases. Fueron realizados trabajos de campo para que los alumnos pudieran conocer los sindicatos de los trabajadores rurales y las actividades allí desarrollados.

- Observaron también que muchos de estos estudiantes tenían vergüenza de decir que vivían y trabajaban en el campo, lo que exigió mucho tiempo para modificar el sentimiento de inferioridad en relación a aquellos que viven en la ciudad. Este trabajo de identidad surtió efectos positivos y, después de algún tiempo, pasaron a tener otra opinión del campo, a valorar el trabajo de sus padres, sus ancestrales, la tierra y los productos que cultivan.

- También criticaron la metodología aplicada en las escuelas, que no tiene relación con el campo. A pesar de trabajar con campesinos, no se hace un trabajo de base, de escucha y diálogo horizontal con la comunidad. Además y se realizan pocos trabajos prácticos para observar in loco lo que se aprende en los libros didácticos.

5 Otras experiencias de prácticas en Tempo Comunidad

- Posibilitaron conocer mejor el municipio donde viven, interactuar con campesinos de diferentes contextos, comprender sus necesidades, deseos, sueños y compartir los propios.

- Tuvieron la oportunidad de conocer y participar del "Consejo de la niñez y adolescencia”, iglesias de la comunidad, asociaciones comunitarias, sindicatos de trabajadores y otros contextos sociales.

- Hicieron ruedas de conversación y círculos de cultura sobre el empoderamiento de las mujeres, división del trabajo, experiencias emprendedoras, trueque con vecinos, producción y procesamiento de mandioca, cacao, entre otros productos típicos regionales. También se habló de medio ambiente, ecología, plantas nativas de la región, cerca viva, sustentabilidad, identidad campesina, cuidados con la tierra, agricultura familiar, productos orgánicos, entre otras cuestiones interesantes para la comunidad.

\section{CONCLUSIÓN}

Como es posible notar, el grado de Educación del Campo posee una propuesta muy distinta a la de otras carreras ofrecidas en la universidad, especialmente porque posee en su propuesta curricular y político pedagógica una intención de establecer una relación más horizontal, dinámica y orgánica con las 
comunidades campesinas a las que pertenecen estudiantes, proponiendo actividades de intervención en sus contextos inmediatos.

La Educación del Campo, desde una perspectiva crítica, ofrece a los estudiantes una infinidad de temáticas relacionas al universo rural, posibles de ser discutidas en las comunidades, para proporcionar un encuentro con su historia, sus raíces y su identidad, creando así un sentimiento de pertenencia que conduzca a un cuidado consigo mismo, con el otro y con la tierra.

De esta forma, al apropiarse de los conocimientos en un ambiente democrático, van progresivamente formándose como ciudadanos en el sentido amplio del término y movilizando esos conocimientos en su cotidiano, ampliando la contribución de la universidad a espacios sociales históricamente marginalizados.

Como fue visto a lo largo de este trabajo, la UFRB, al estar sintonizada con su contexto local y comprometida con la movilización del conocimiento, ofrece una gran contribución en ese proceso de toma de consciencia colectiva. Eso demuestra su propuesta contra hegemónica, única capaz de hacer frente al agro negocio y sus implicaciones en el campo.

Para tanto, es importante que los profesores estimulen y orienten a los estudiantes a prepararse, articularse, participar de círculos de cultura, congresos y debates para servir, aún mejor, a los campesinos de las comunidades donde viven. Al hacer un puente entre la universidad y la comunidad, contribuyen en el proceso de formación de ciudadanos, capaces de cuestionar, discutir, criticar, proponer y presionar al sistema para crear las condiciones fundamentales de desarrollo de sus territorios y garantizar los medios estructurales para que todos puedan vivir con dignidad en el lugar que eligieron. Rescatar ese horizonte utópico es fundamental para movilizar a la sociedad para que realice cambios estructurales más substanciales y duraderos.

Finalmente, esta temática puede y debe seguir siendo investigada, porque se trata de un campo fértil para nuevas contribuciones. Así, es nuestro deseo que los resultados de este trabajo puedan provocar discusiones en torno de estas cuestiones y proporcionar a los interesados un material a ser leído y aprovechado para servir de estímulo a nuevas iniciativas.

\section{REFERENCIAS BIBLIOGRÁFICAS}

BARRETO, Itana Augusta. Cartografia de discursos e práticas como processo pedagógico: Um estudo sobre educação Ambiental no Ingá. Monografia do Curso de Especialização em Educação Ambiental para Sustentabilidade. Feira de Santana: UEFS, 2012.

FREIRE, Paulo. Pedagogia da autonomia: saberes necessários à prática pedagógica. $13^{\mathrm{a}}$ ed. São Paulo: Paz e Terra, 1996.

FREIRE, Paulo. Pedagogia da indignação: cartas pedagógicas e outros escritos. São Paulo: UNESP, 2000.

FREITAS, K. Uma Inter-relação: políticas públicas, gestão democratico-participativa na escola pública e formação da equipe escolar. Em Aberto, Brasília, v. 17, n. 72, fev./jun, 47-59, 2000.

IBGE. (2010). Instituto Brasileiro de Geografia e Estatística: Censo 2010. - disponible en: $<$ http://cidades.ibge.gov.br/xtras/uf.php?lang=\&coduf=29\&search=bahia> acceso en 23 de mayo de 2019.

NAIDORF, Judith. Los cambios en la cultura académica de la universidad pública. $1^{\text {a }}$ ed. Buenos Aires: Eudeba, 2009.

NAIDORF, Judith; PERROTTA, Daniela. La ciencia social politizada y móvil de una nueva agenda sulamericana orientada a prioridades. Revista de la Educación Superior. Vol. XLIV. No 174, abril-junio de 2015. Mexico: ANUIES, p. 19-46, 2015. 
NAIDORF, Judith; SANDES, André B.. Utilidad, aplicabilidad y movilización del conocimiento: un estudio de caso del curso de Licenciatura en Educación del campo de la Universidad Federal do Recôncavo de Bahia-Brasil. Estudios Rurales, Buenos Aires, v. 9, n. 17, p. 37-63, 2019.

SANDES, André B. A cultura da depredação do ônibus escolar: a educação formal começa no caminho para escola. Textura, Governador Mangabeira-BA, v. 13, n. 21, p. 029-040, jan - jun, 2019.

SANTOS, Fábio Josué Souza dos. Histórico do Curso de Mestrado Profissional em Educação do Campo da UFRB. Amargosa-Ba: UFRB; Programa de Pós-Graduação em Educação do Campo, 17 de fevereiro de 2018. (Digitado. Texto não publicado), 2018.

SAVIANI, Dermeval. Pedagogia Histórico-Crítica. 8ª ed. Campinas, São Paulo: Autores Associados, 2003. 